\title{
Analysis of gene evolution and metabolic pathways using the Candida Gene Order Browser
}

\author{
David A Fitzpatrick*1,2, Peadar O'Gaora³, Kevin P Byrne ${ }^{4}$ and Geraldine Butler*1
}

\begin{abstract}
Background: Candida species are the most common cause of opportunistic fungal infection worldwide. Recent sequencing efforts have provided a wealth of Candida genomic data. We have developed the Candida Gene Order Browser (CGOB), an online tool that aids comparative syntenic analyses of Candida species. CGOB incorporates all available Candida clade genome sequences including two Candida albicans isolates (SC5314 and WO-1) and 8 closely related species (Candida dubliniensis, Candida tropicalis, Candida parapsilosis, Lodderomyces elongisporus, Debaryomyces hansenii, Pichia stipitis, Candida guilliermondii and Candida lusitaniae). Saccharomyces cerevisiae is also included as a reference genome.

Results: CGOB assignments of homology were manually curated based on sequence similarity and synteny. In total CGOB includes 65617 genes arranged into 13625 homology columns. We have also generated improved Candida gene sets by merging/removing partial genes in each genome. Interrogation of CGOB revealed that the majority of tandemly duplicated genes are under strong purifying selection in all Candida species. We identified clusters of adjacent genes involved in the same metabolic pathways (such as catabolism of biotin, galactose and $\mathrm{N}$-acetyl glucosamine) and we showed that some clusters are species or lineage-specific. We also identified one example of intron gain in C. albicans.
\end{abstract}

Conclusions: Our analysis provides an important resource that is now available for the Candida community. CGOB is available at http://cgob.ucd.ie.

\section{Background}

Fungal infections are the fourth most common nosocomial bloodstream infection in the United States. Candida species account for approximately $10 \%$ of all bloodstream infections [1] and worldwide are the most common cause of opportunistic fungal infection [2]. Due to their increasing clinical importance, recent sequencing projects have determined the complete sequence of ten Candida genomes, including common pathogenic species and species rarely, if ever, associated with disease [3-7].

The term Candida was originally assigned to imperfect yeast species, with no known sexual cycle. This term now covers a variety of species of diverse origins (both sexual and asexual), and provides little information regarding

\footnotetext{
*Correspondence: david.fitzpatrick@nuim.ie, geraldine.butler@ucd.ie 1 UCD School of Biomolecular and Biomedical Science, Conway Institute, University College Dublin, Belfield, Dublin 4, Ireland
}

Full list of author information is available at the end of the article evolutionary relationships. For example, Candida glabrata is more closely related to Saccharomyces cerevisiae than it is to Candida albicans. Debaryomyces hansenii and Pichia stipitis are close relatives of Candida species [8]. Some species, such as C. lusitaniae, were assigned two names, one (Candida lusitaniae) referring to the asexual (anamorph) form, and one (Clavispora lusitaniae) to the sexual (teleomorph) form. Similarly, Candida guilliermondii is also known as Pichia guilliermondii, and Candida famata as D. hansenii. These species share a relatively recent common ancestor (Figure 1 ), and in all cases the codon CUG is translated as serine rather than leucine [9]. For brevity, we refer to the above as Candida species that belong to the CTG clade $[5,8,10]$.

Previous comparative analysis of eight Candida genomes led to the identification of gene families that are highly represented in strongly pathogenic species (such as C. albicans, C. tropicalis, C. parapsilosis), compared to weak pathogens such as C. lusitaniae and C. guilliermon$d i i$, and very rare or non-pathogenic species such as $D$. 


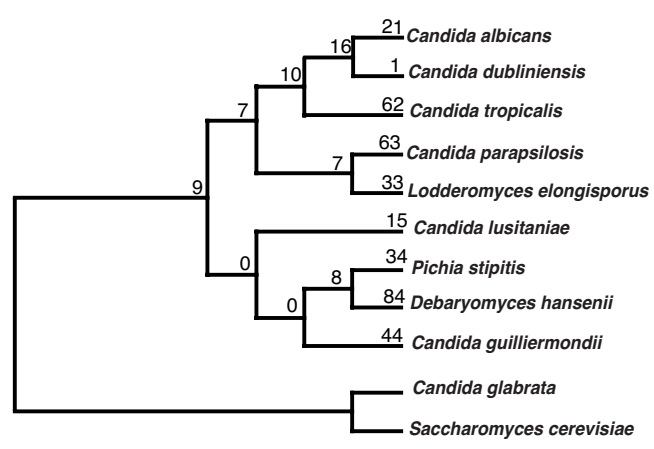

Figure 1 Phylogenetic supertree of Candida species represented in CGOB. Candida glabrata and Saccharomyces cerevisiae have been selected as outgroups. Numbers on branches represent tandem duplications gained along each lineage.

hansenii [5]. These include three cell wall families; the ALS-like adhesins, which in C. albicans have been associated with virulence, biofilm development and acquisition of iron from the host [11-13]; the Pga30-like family [14], and the Hyr/Iff family [15]. Many families are highly enriched for gene duplications [5].

There are currently two major browsers that display Candida genomes, CandidaDB, which contains information for several Candida genomes, and the Candida Genome Database, which predominantly describes $C$. albicans $[16,17]$. The major disadvantage of these browsers is that it is difficult to compare genomes to each other. They also display a to-scale representation of a chromosomal region, which is unsuitable for analysis of gene order and evolution. To overcome these problems we developed the Candida Gene Order Browser (CGOB [18]).

CGOB incorporates all available genome sequences from Candida species, including two isolates of C. albicans (SC5314 and WO-1), its close relative and minor pathogen $C$. dubliniensis, the major pathogens C. tropi- calis and C. parapsilosis, the minor pathogens $L$. elongisporus, C. lusitaniae and C. guilliermondii, the marine yeast $D$. hansenii, and $P$. stipitis, a xylose-digesting yeast that is associated with beetles found in wood [3-7]. CGOB is based on the engine developed for the Yeast Gene Order Browser (YGOB) $[19,20]$, which has been applied to the analysis of genome duplication in the Saccharomyces group. To construct CGOB, all assignments of homology were manually curated, based on sequence similarity and gene order (synteny). Partial genes in each genome were identified and removed, leading to the generation of improved gene sets. CGOB was then used to analyze gene duplication, intron localization and clustering of genes involved in metabolic pathways. We found that the majority of tandemly duplicated genes are under strong purifying selection and that there are both conserved and species-specific clusters of metabolically related genes in Candida. CGOB is available at http:// cgob.ucd.ie.

\section{Results and discussion}

CGOB structure and Candida genome editing

Version 1 of CGOB includes ten Candida genomes obtained from a variety of sequencing centers (Table 1), together with the genome from S. cerevisiae. CGOB's visual display consists of horizontal tracks representing chromosomal segments and pillars (Figure 2). Pillars are the core data structures used to store list of homologies across all species represented in the gene order browser [20]. Pillars contain vacant slots when homologous genes cannot be found in a particular genome. Genes were initially added to pillars based on automated assignments derived from best bidirectional BLASTP searches. The CGOB pillar dataset was manually refined by examining regions of dubious synteny and singleton genes. A combination of BLASTP scores, synteny and phylogenetic data were used to confirm assignments to pillars.

Table 1: Candida species displayed in CGOB.

\begin{tabular}{lccccc}
\hline \multicolumn{1}{c}{ Species } & Citation & Genes & Partial ORFs & Refined Gene set & Singletons \\
\hline C. albicans SC5314 & {$[6]$} & 6,185 & 0 & 6,185 & 43 \\
C. albicans WO-1 & {$[5]$} & 6,197 & 91 & 6,148 & 99 \\
C. dubliniensis CD36 & {$[7]$} & 5,924 & 0 & 5,924 & 200 \\
C. tropicalis MYA-3404 & {$[5]$} & 6,258 & 116 & 6,198 & 737 \\
C. parapsilosis CDC 317 & {$[5]$} & 5,823 & 28 & 5,809 & 553 \\
L. elongisporus NRLL YB-4239 & {$[5]$} & 5,802 & 173 & 5,710 & 596 \\
P. stipitis CBS6054 & {$[3]$} & 5,838 & 12 & 5,832 & 470 \\
D. hansenii CBS767 & {$[4]$} & 6,317 & 12 & 6,311 & 981 \\
C. guilliermondii ATCC6260 & {$[5]$} & 5,920 & 142 & 5,844 & 666 \\
C. lusitaniae ATCC 42720 & {$[5]$} & 5,941 & 135 & 5,869 & 881 \\
\hline
\end{tabular}




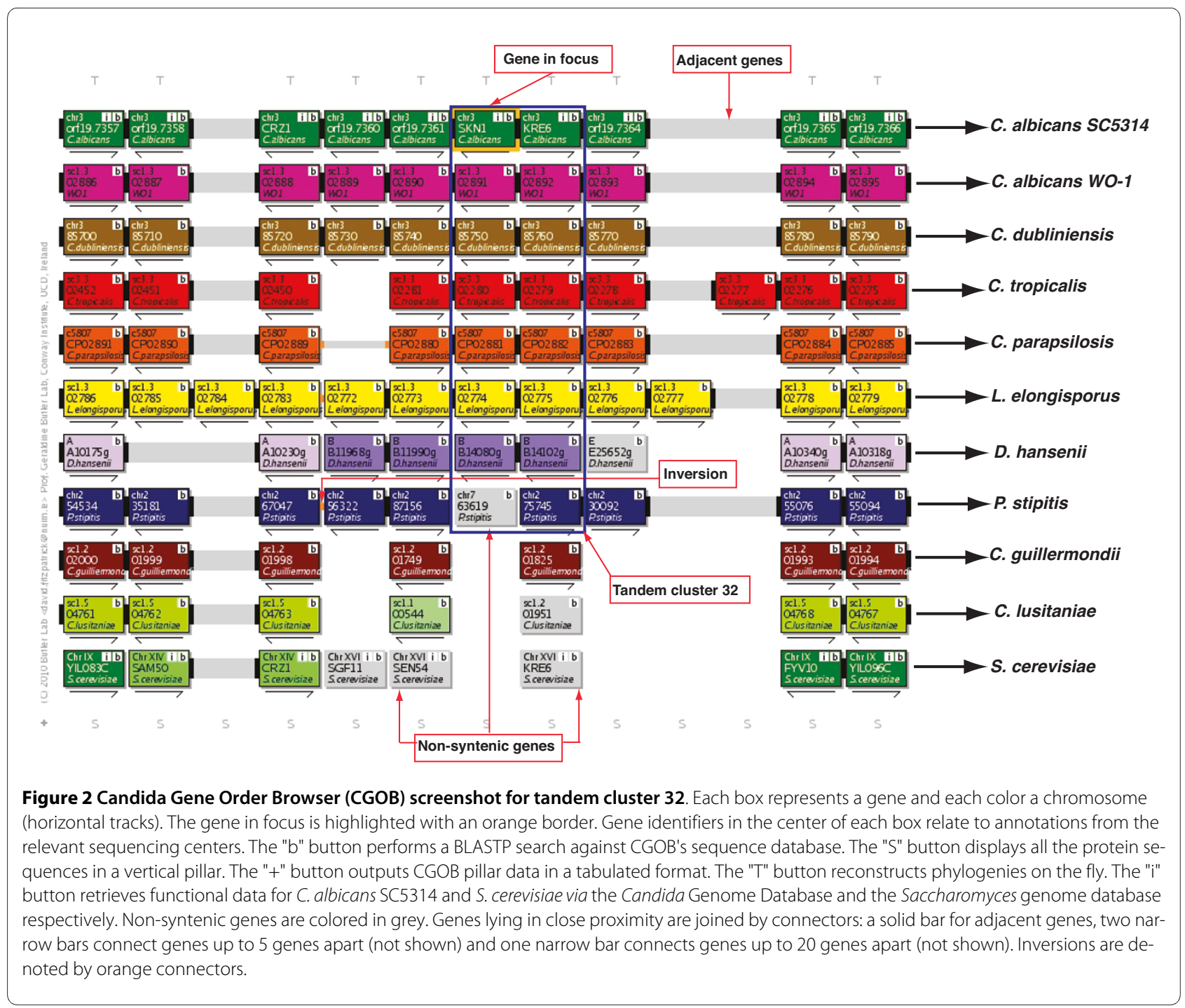

Similar to YGOB, CGOB allows the user to focus the screen display on a gene of interest and to view phylogenetic trees, sequences and BLASTP results (Figure 2). Hyperlinks to functional data can also be accessed for C. albicans SC5314 and S. cerevisiae via the Candida Genome Database [17] and the Saccharomyces Genome Database [21] respectively.

During manual editing of CGOB we observed that the original genome annotations contained a substantial number of apparently partial open reading frames, which we merged into full-length gene models. For example, LELG_01495 and LELG_1496 in L. elongisporus are both similar to parts of $C$. albicans orf19.6045 (PSD1). Closer inspection showed that LELG_1496 aligned with the Nterminus of orf19.6045 whereas LELG_1496 matches the C-terminus (Additional file 1). In cases like this we deleted the partial open reading frames from CGOB's homology pillars and inserted a new "merged" gene model. Overall, we identified 709 ORFs in 8 genomes that were subsequently merged into 335 full-length genes (Additional file 2). The L. elongisporus genome contained the highest number of partial ORFs (173 in total), while the highly curated genomes of C. albicans SC5314 and C. dubliniensis contain none (Table 1). The corrected gene sets are available for download from CGOB [18].

\section{Tandem Gene Duplications}

Tandem gene duplication is one mechanism by which species acquire new genes, and by extrapolation, new functions. We therefore used both similarity and synteny measurements in CGOB to identify gene duplications in all Candida genomes. We identified and numbered all tandem clusters in each genome (Additional file 3). For some rapidly evolving genes, sequence similarity is not high enough to identify family members. For example, our initial BLAST based approach suggested that orf19.2508 (PRM9) and orf19.2509 in C. albicans are tandem duplicates (cluster 30, Additional file 3). Their 
orthologs in C. dubliniensis, C. tropicalis, C. parapsilosis and $L$. elongisporus are found adjacent to one another. However, these genes were not initially identified as tandem duplicates in the other species because they do not have a BLASTP $E$-value below our initial cut-off (See Methods). Therefore, slower evolving tandem duplicates in one Candida species (C. albicans SC5314 in this example) can be used to locate rapidly evolving tandems (or tandems with low sequence complexity) in other Candida genomes.

Tandem duplicates that subsequently underwent chromosomal rearrangement are difficult to identify. However, the ancestral arrangement can be inferred from an analysis of homologous genes in CGOB. For example, in C. albicans SC5314, the duplicate genes orf 19.7362 (SKN1) and orf19.7363 (KRE6) are located beside one another on chromosome 3 (Figure 2, Additional file 3 (cluster 32)). Their orthologs in C. albicans WO-1, C. dubliniensis, C. tropicalis, C. parapsilosis, L. elongisporus and $D$. hansenii are also adjacent (Figure 2). However in $P$. stipitis, SKN1 (PICST_63619) is located on chromosome 7 while KRE6 (PICST_75745) is located on chromosome 2 (Figure 2). The most parsimonious explanation is that a duplication of SKN1 or KRE6 occurred in an ancestor of all the Candida species, and this has been conserved in most. However, relocation of SKN1 has occurred exclusively in P. stipitis (Figure 2).

In total 901 tandem clusters were identified across all the Candida genomes (Table 2, Additional file 3). C. lusitaniae has the smallest number of tandem clusters (44) whereas C. parapsilosis has the highest number (139). This is noticeably high, as the closest relative of C. parapsilosis, L. elongisporus (Figure 1), contains only 93 tandem clusters (Table 2). The average number of genes per tandem cluster in all Candida species ranges is slightly greater than 2 (Table 2).
We used CGOB to map species and lineage-specific tandem duplications (Figure 1). For example, since they last shared a common ancestor, $C$. albicans has undergone at least 21 species-specific tandem duplications gaining 24 paralogs, while its close relative $C$. dubliniensis has undergone a single tandem duplication (Cd36_11890, Cd36_11900) gaining 1 additional gene (Additional file 3, cluster 463). Similarly, C. parapsilosis has undergone 63 species-specific tandem duplications gaining 78 paralogs since diverging from its closest relative, L. elongisporus, which has undergone 33 tandem duplication gaining 41 paralogs in the same time (Additional file 3).

Cluster 22 (Additional file 3) illustrates an ancient duplication, resulting in a family of peroxisomal acylCoA thioesterases that are present in 3-5 tandem copies in all the Candida species. The cluster is particularly large in the branch containing C. albicans, C. dubliniensis, C. tropicalis, C. parapsilosis and L. elongisporus, where 5 family members are immediately adjacent to each other. The single homolog in S. cerevisiae is likely to be involved in fatty acid oxidation [22]. There is significant up-regulation of fatty acid $\beta$-oxidation when C. albicans cells are engulfed by macrophages [23], although this pathway does not appear to be essential for virulence [24]. Many of the other tandem duplication clusters include members of larger gene families, such as lipases (cluster 10, Additional file 3), glucose transporters (cluster 15,61, Additional file 3) and ferric reductases (cluster 57, Additional file 3). Some clusters are lineage specific, such as the triplication of the pirin-domain genes PRN2, PRN3 and PRN4 (cluster 6, Additional file 3) in C. albicans, C. dubliniensis and C. tropicalis. The function of these genes in unknown, but they are likely to localize to the nucleus. There is an amplification of the FRP6 family in D. hansenii and P. stipitis; the S. cerevisiae orthologs are required for export of ammonia [25] (cluster 497, Additional file 3). Other clusters are species-specific,

Table 2: The total number of tandem duplicates found in each Candida species displayed in CGOB.

\begin{tabular}{|c|c|c|c|c|c|}
\hline Species & $\begin{array}{l}\text { Tandem } \\
\text { Clusters }\end{array}$ & $\begin{array}{c}\text { Species specific } \\
\text { clusters }\end{array}$ & $\begin{array}{l}\text { \# of genes } \\
\text { in clusters }\end{array}$ & $\begin{array}{c}\text { Average \# of } \\
\text { genes per cluster }\end{array}$ & $\begin{array}{c}\text { Tandem } \\
\text { Duplicates }\end{array}$ \\
\hline C. albicans & 106 & 21 & 230 & 2.17 & 124 (1.99\%) \\
\hline C. dubliniensis & 83 & 1 & 179 & 2.16 & $96(1.62 \%)$ \\
\hline C. tropicalis & 125 & 62 & 284 & 2.27 & $159(2.56 \%)$ \\
\hline C. parapsilosis & 139 & 63 & 328 & 2.36 & $189(3.25 \%)$ \\
\hline L. elongisporus & 93 & 33 & 206 & 2.20 & $114(1.99 \%)$ \\
\hline D. hansenii & 132 & 84 & 294 & 2.23 & $162(2.56 \%)$ \\
\hline P. stipitis & 96 & 40 & 204 & 2.12 & $108(1.85 \%)$ \\
\hline C. guilliermondii & 83 & 44 & 181 & 2.18 & $98(1.67 \%)$ \\
\hline C. Iusitaniae & 44 & 15 & 97 & 2.20 & $53(0.90 \%)$ \\
\hline
\end{tabular}

Percentages in parenthesis refer to the total percentage of the genome that has arisen through tandem duplication. 
such as the five adjacent 2' hydroxyisoflavone reductases (CIP1) described by Jeffries and Van Vleet [26], which our analysis confirms is unique to P. stipitis (cluster 228). Most of the other species have a single copy, except for $C$. parapsilosis, which has two. L. elongisporus contains 5 tandem repeats of a large family with up to 13 members in this species, which is absent from all the other Candida genomes (cluster 280, Additional file 3). The function of this family is unclear but all members contain a Phosphatidylinositol Phosphate Kinase (PIPKc) domain.

We also determined whether tandem duplicates in individual Candida species are undergoing positive selection. Recent genome wide studies have shown that positive selection after tandem duplication can give rise to novel gene functions [27], that may help pathogens evade the human immune response [28]. At the DNA level, positive selection may be detected by comparing the rate of amino acid altering (nonsynonymous) nucleotide substitutions with the rate of synonymous substitution $\left(\mathrm{d}_{\mathrm{N}} / \mathrm{d}_{\mathrm{S}}\right)$. A $\mathrm{d}_{\mathrm{N}} / \mathrm{d}_{\mathrm{S}}$ ratio $>1$ is indicative of positive selection. The average $\mathrm{d}_{\mathrm{N}} / \mathrm{d}_{\mathrm{S}}$ ratio for all tandem clusters was found to be 0.27 (not shown). Of the 901 Candida clusters examined, only 12 displayed a $d_{N} / d_{S}$ ratio $>1$ (Additional file 3). Five of these are species-specific and have no homologs in any other Candida species (or any species in GenBank). The remaining 7 clusters under the influence of positive selection do not share homology with gene families (cell wall, hyphal, pseudohyphal, filamentous growth and biofilm functions) normally associated with pathogenicity in Candida [5]. In P. stipitis, one cluster encodes putative ubiquitin protein ligases, one encodes zinc finger-containing proteins, and one encodes potential siderophore transporters (Additional file 3). In D. hansenii, one cluster encodes orthologs of TFS1, whose expression is induced during filamentation in C. albicans [29]. Overall our results suggest that the majority of Candida tandem duplicates are under the influence of strong purifying selection, presumably to conserve gene function.

We have extended an earlier analysis of duplicate genes in Candida genomes, which considered only members of multigene families [5]. We also identified some clusters by manual inspection. For example, we first identified 85 tandem clusters in C. albicans SC5314 using a simple BLAST approach, and this was increased to 106 using synteny information, whereas only 24 were reported in Butler et al [5]. In some species (such as L.elongisporus and C. guilliermondii) we identified a smaller number of clusters than Butler et al [5], partly because we removed partial ORFs from the gene sets.

\section{The Candida Paranome}

Using our reannotated Candida genomes we determined the number of multigene families (the paranome [30]) for each Candida species. C. tropicalis has the highest number (557), whereas C. lusitaniae (390) has the lowest (Table 3). In contrast, C. parapsilosis has the lowest number (4377) of genes that do not belong to families, whereas C. lusitaniae (4871) has the highest (Table 3). The average number of genes per multigene family is approximately 3 for all species, although all Candida species have larger gene families (Table 3).

The largest gene family shared by all species contains a transporter (DIP5) annotated as a putative dicarboxylic amino acid permease in CGD. This family was previously suggested as a potential antifungal target, as there are no

Table 3: The Candida paranome.

\begin{tabular}{|c|c|c|c|c|c|c|c|}
\hline & Unique genes & $\begin{array}{l}\text { Multigene } \\
\text { Families }\end{array}$ & $\begin{array}{c}\text { Average \# genes } \\
\text { per family }\end{array}$ & \multicolumn{4}{|c|}{ Gene families containing } \\
\hline $\begin{array}{l}\text { C. albicans } \\
\text { SC } 5314\end{array}$ & $4662(76.3 \%)$ & 484 & 2.99 & $10.0 \%$ & $3.9 \%$ & $2.7 \%$ & $7.1 \%$ \\
\hline C. albicans W01 & $4556(76.8 \%)$ & 467 & 2.95 & $10.3 \%$ & $3.4 \%$ & $2.7 \%$ & $6.8 \%$ \\
\hline C. dubliniensis & 4449 (75.1\%) & 513 & 2.87 & $11.4 \%$ & $3.8 \%$ & $3.0 \%$ & $6.7 \%$ \\
\hline C. tropicalis & $4603(73.6 \%)$ & 557 & 2.97 & $11.8 \%$ & $3.7 \%$ & $2.6 \%$ & $8.3 \%$ \\
\hline C. parapsilosis & $4377(75.2 \%)$ & 472 & 3.06 & $10.5 \%$ & $3.5 \%$ & $2.4 \%$ & $8.4 \%$ \\
\hline L. elongisporus & $4616(79.6 \%)$ & 413 & 2.86 & $9.3 \%$ & $3.6 \%$ & $2.2 \%$ & $5.3 \%$ \\
\hline P. stipitis & 4335 (74.3\%) & 497 & 3.02 & $10.5 \%$ & $4.6 \%$ & $2.8 \%$ & $7.8 \%$ \\
\hline C. guillermondii & 4558 (77.0\%) & 473 & 2.87 & $10.5 \%$ & $4.5 \%$ & $1.6 \%$ & $6.4 \%$ \\
\hline C. Iusitaniae & $4871(82.0 \%)$ & 390 & 2.74 & $9.3 \%$ & $3.0 \%$ & $1.6 \%$ & $4.1 \%$ \\
\hline
\end{tabular}

The number of single- and multi- gene families in each Candida species displayed in CGOB. 
homologs in humans [31]. All Candida species have at least 20 members of this family (not shown). The $M E P$ family, encoding three ammonium permeases in C. albicans SC5314, has also suggested as an antifungal drug target [31]. Three $M E P$ genes are present in all Candida species except for L. elongisporus, which is missing the ortholog of one (orf19.4446, not shown). Drugs directed against these families should therefore be of broad specificity and target all Candida species, and are likely to have no undesired interactions with the human patient.

Approximately 20-25\% of all Candida genes in CGOB belong to a multigene family (Table 3), similar to what has previously been reported for C. albicans SC5314 [31]. This figure is lower than what has been observed for $S$. cerevisiae ( $30 \%)$, which is unsurprising as S. cerevisiae has undergone a whole genome duplication [32] while Candida species have not [33].

\section{Intron loss in Candida genes}

Yeast genomes from the Saccharomycotina are known to be intron poor; introns are found in fewer than $5 \%$ of genes from most species [34]. The exact mechanisms of intron loss are not fully elucidated, but it is likely to occur via recombination of a chromosomal copy with a reverse transcript $[35,36]$. Introns have been predicted with some accuracy in only three of the sequenced Candida genomes - C. albicans, C. dubliniensis and D. hansenii. We therefore restricted our analysis to these species. Where we observed differences in intron locations in tandem duplicates in any one species, the corresponding genomic sequence of the other two was manually inspected to confirm intron presence or absence.

C. albicans SC5314 has at least 381 genes containing 415 introns [37]. Of these genes, $79(\sim 21 \%)$ belong to a multigene family, and five are located in tandem clusters.

Cluster 5 contains three paralogs in C. albicans (orf19.5194.1, orf19.6837 (FMA1), and orf19.6838) (Additional file 3 and Figure $3 \mathrm{~A})$. The first two of these genes contain introns, as do their orthologs in C. dubliniensis. There is a single homolog in $D$. hansenii, which has undergone an inversion relative to $C$. albicans, but still contains an intron. However, the third gene (orf19.6838) does not contain an intron in any of the species (Figure $3 \mathrm{~A})$. The most likely hypotheses are that either the progenitor copy contained an intron and was duplicated twice, followed by intron loss in one copy, or that the progenitor was first duplicated to generate a second introncontaining copy, and then duplicated again in an RNAmediated event.

Cluster 144 (Additional file 3) contains members of a family of glutathione-S-transferases that are present in two adjacent copies in some species and three in others (Figure 3B). Two genes in C. albicans, C. dubliniensis and $D$. hansenii have introns, suggesting that they arose

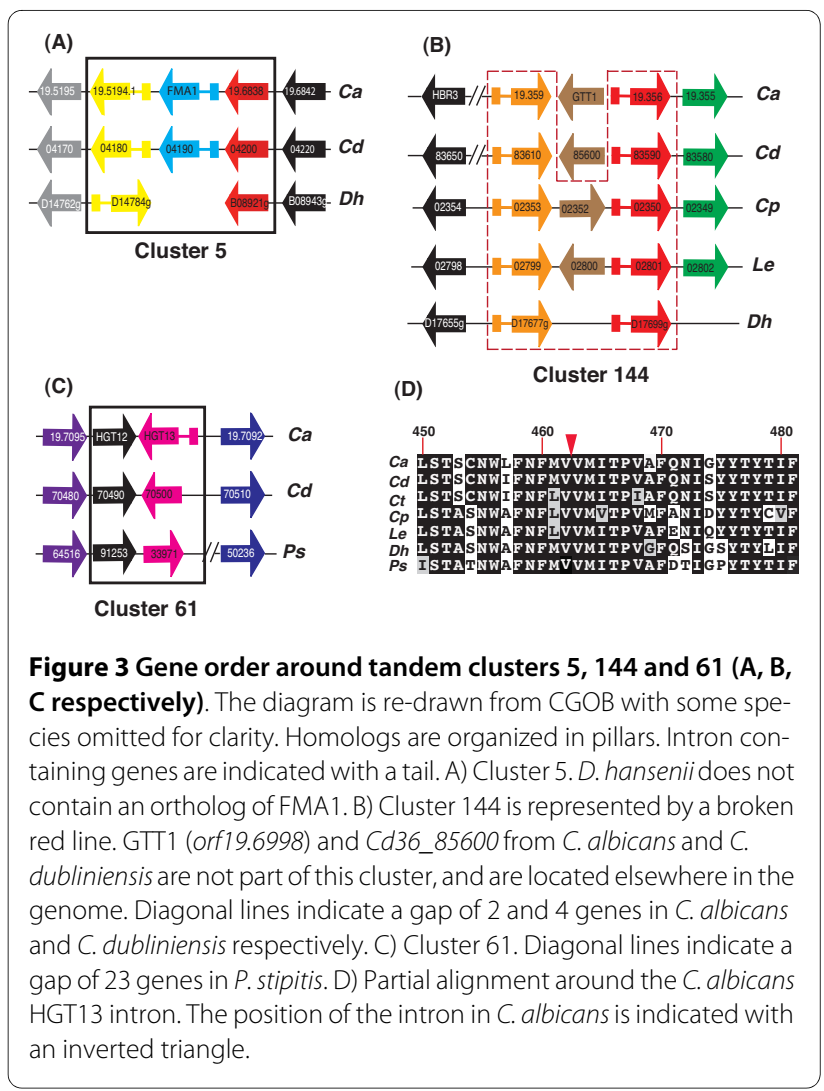

through tandem duplication (Figure 3B). However, a third member of the family (GTT1) which lies within the cluster in C. parapsilosis, L. elongisporus and C. guilliermondii does not contain any introns in $C$. albicans or $C$. dubliniensis (there is no ortholog in D. hansenii). GTT1 may therefore have arisen via an RNA intermediate in an ancestral Candida species.

We also found evidence for a species-specific intron gain in a tandem duplicate. Cluster 61 (Additional file 3) contains two adjacent genes (HGT12 and HGT13) in C. albicans, $C$. dubliniensis and $P$. stipitis that belong to a large family of sugar transporters that have at least 20 members in Candida species [38] (Figure 3C). The HGT13 homolog is not adjacent to HGT12 in the other species (not shown). In C. albicans, HGT13 contains an intron, whereas its paralog HGT12 does not. The HGT13 intron lies within the coding sequence, which makes it easy to identify (Figure 3D). Interestingly, although this intron is present in HGT13 from both C. albicans isolates, it is absent from all of the homologs in the other species, whether syntenic or not (Figure 3C). Intron gain is very rare [36,39], but it appears more likely that HGT13 in C. albicans gained an intron, rather than the intron was independently lost from all the other species. At least one other member of the HGT family (HGT9) also contains introns in C. albicans, but these appear to be conserved in C. dubliniensis only. 
Table 4: CGD metabolic pathways that show evidence of gene clustering.

\begin{tabular}{|c|c|c|c|c|c|c|c|c|c|c|c|}
\hline & \#Genes & SC5314 & W01 & Cdub & Ctro & Cpar & Lelo & Dhan & Psti & Pgui & Clus \\
\hline $\begin{array}{l}\text { Histidine, purine and pyrimidine } \\
\text { biosynthesis }\end{array}$ & 41 & 7 & 7 & 7 & 5 & 4 & 4 & - & 3 & 4 & - \\
\hline Aerobic respiration (cyanide sensitive) & 14 & 3 & 3 & 3 & 3 & 2 & 3 & 3 & 3 & 2 & 2 \\
\hline Aerobic respiration (cyanide insensitive) & 8 & 2 & 2 & 2 & 2 & 2 & - & - & - & - & - \\
\hline 2-keto glutarate dehydrogenase complex & 3 & 2 & 2 & 2 & 2 & - & - & - & 2 & - & - \\
\hline $\mathrm{N}$-acetylglucosamine degradation & 3 & 3 & 3 & 3 & 3 & 3 & 3 & 3 & 3 & 3 & 3 \\
\hline Methylglyoxal pathway & 12 & 2 & 2 & 2 & 2 & 2 & 2 & - & - & - & - \\
\hline Sphingolipid metabolism & 8 & - & - & - & - & - & - & - & - & 2 & - \\
\hline Ergosterol biosynthesis & 21 & - & - & - & - & - & - & 2 & - & 2 & 2 \\
\hline Biotin biosynthesis & 4 & 4 & 4 & - & 3 & - & - & 2 & - & - & - \\
\hline NAD salvage pathway & 4 & - & - & - & 2 & - & - & - & - & - & - \\
\hline Tetrapyrrole biosynthesis & 4 & 2 & 2 & 2 & 2 & - & - & - & 2 & - & - \\
\hline Pantothenate and coA biosynthesis & 11 & 2 & 2 & 2 & 2 & 2 & 2 & 2 & 2 & 2 & 2 \\
\hline Starch degradation & 7 & - & - & - & - & - & - & - & - & - & 2 \\
\hline Lipid-linked oligosaccharide biosynthesis & 8 & - & - & - & 2 & 2 & 3 & 2 & 3 & 2 & - \\
\hline Arginine degradation (arginase pathway) & 5 & 2 & 2 & 2 & - & - & - & 2 & 2 & 2 & 2 \\
\hline Lysine biosynthesis & 6 & - & - & - & 2 & - & - & - & - & - & - \\
\hline Superpathway of glycine biosynthesis & 5 & - & - & - & - & 2 & - & - & - & - & - \\
\hline Biosynthesis of phe/tyr/trp & 13 & - & - & - & - & - & 2 & - & - & - & - \\
\hline Superpathway of glycine biosynthesis & 5 & - & - & - & - & 5 & - & - & - & - & - \\
\hline Acrylonitrile degradation & 4 & - & - & - & $2^{(2 T)}$ & $2^{(2 T)}$ & $2^{(2 T)}$ & - & $2^{(2 \mathrm{~T})}$ & - & - \\
\hline Galactose degradation & 5 & $4(2 D)$ & $4(2 D)$ & $4(2 D)$ & $4(2 D)$ & $4(2 D)$ & $4(2 D)$ & $4(2 D)$ & $4(2 D)$ & $4(2 D)$ & $4(2 D)$ \\
\hline tRNA charging pathway & 35 & $13^{(6 \mathrm{D})}$ & $13^{(6 \mathrm{D})}$ & $13^{(6 D)}$ & $10^{(2 \mathrm{D})}$ & $7^{(4 D)}$ & $9^{(6 D)}$ & $9^{(4 D)}$ & $13^{(6 D)}$ & $9^{(4 D)}$ & $5^{(4 \mathrm{D})}$ \\
\hline Fatty acid oxidation pathway & 14 & $4^{(4 D)}$ & $4^{(4 D)}$ & $2^{(2 D)}$ & $4^{(4 D)}$ & $4^{(4 D)}$ & - & $2^{(2 D)}$ & $2^{(2 D)}$ & $2^{(2 T)}$ & $2^{(2 \mathrm{~T})}$ \\
\hline Glutathione-glutaredoxin redox reactions & 9 & $5^{(3 D, 3 D)}$ & $5(2 \mathrm{D}, 2 \mathrm{~T}) *$ & $6^{(3 D, 3 T)}$ & $3^{(3 T)}$ & $4^{(4 T)}$ & $3^{(3 T)}$ & $2^{(2 T)}$ & $2^{(2 T)}$ & $3^{(3 \mathrm{~T})}$ & $2^{(2 D)}$ \\
\hline Isoleucine \& phenylalanine degradation & 12 & $2^{(2 \mathrm{~T})}$ & $2^{(2 \mathrm{~T})}$ & - & - & - & - & - & - & - & - \\
\hline Removal of superoxide radicals & 7 & $2^{(2 \mathrm{~T})}$ & $2^{(2 \mathrm{~T})}$ & $2^{(2 T)}$ & - & - & - & - & - & - & - \\
\hline
\end{tabular}

Superscript numerals in parenthesis refer to the numbers of genes that are either paralogs ${ }^{\mathrm{D}}$ or tandem duplicates ${ }^{\mathrm{T}}$.

Other gene families that are not tandemly arranged are also likely to have arisen through both DNA-based gene duplication and via an RNA intermediate, possibly including retrotransposition. For example in C. albicans, the glycosylphosphatidylinositol-linked cell wall gene ECM33 [40] has two paralogs orf19.4955 and orf19.4255 (ECM331) that are not adjacent to each other (not shown). ECM33 and orf19.4955 both contain an intron, as do their orthologs in C. dubliniensis and D. hansenii. ECM331 does not contain an intron in any of the three species. This suggests that ECM33 and orf19.4955 may have arisen through duplication, and ECM331 is the result of reverse transcription of one of the intron-containing paralogs, in an ancestor of the three species.

\section{Clustering of adjacent genes in metabolic pathways}

In bacteria the primary method of controlling gene expression is the organization of genes into operons, which are transcribed into a single mRNA. Bacterial operons often contain genes from the same metabolic pathway. Operons are not usually found in eukaryotes, with the notable exception of nematodes [41-43]. However, there is evidence for clustering of genes at the same genomic location belonging to the same metabolic pathways in fungi. For example, genes involved in secondary metabolism are clustered in the genomes of filamentous ascomycetes [44], and many of the genes involved in metabolism of allantoin and galactose are clustered in the genome of $S$. cerevisiae and related species $[45,46]$. Many functionally-related genes are co-expressed, even when they do not share sequence similarity [26]. Lee and Sonnhammer [47] found that there is significant tendency for genes from the same metabolic pathway to cluster in the genomes of fungi, and in other organisms. However, their definition of proximity was very large, and included genes 
that were separated by up to 400 other genes. Our analysis had a more focused approach, as we searched for evidence of genes involved in the same metabolic pathway lying up to 10 genes apart in Candida species.

Currently there are 155 metabolic pathways that have been manually curated by the Candida Genome Database. However, 23 of these contain only one gene, and a further 33 are redundant. For example, the list of genes involved in the acrylonitrile and aldoxime degradation pathways are identical. Similarly the tyrosol, tryptophan, phenylalanine and chorismate biosynthesis are all subsets of the superpathway of phenylalanine, tyrosine and tryptophan biosynthesis. There are 99 unique pathways, containing 659 genes. There are 511 unique genes in total, representing $8.2 \%$ of the C. albicans SC5314 gene set.

CGOB was interrogated for evidence of clustering of genes (i.e. lying within 10 genes of one another on the same chromosome) in the 99 nonredundant pathways. We identified 21 pathways that display evidence of gene clustering in at least one Candida species (Table 4 and Additional file 4). Some metabolic pathway clusters result from tandem duplication; for example, $A O X 1$ and $A O X 2$ (encoding cyanide insensitive enzymes required for an alternative pathway of aerobic respiration) in C. albicans, C. dubliniensis, C. tropicalis and C. parapsilosis, were also identified as tandem cluster 9 (Additional file 3). There is evidence of species-specific clusters of unrelated genes, such as lysine biosynthesis and glycine biosynthesis, which are clustered in one species only (C. tropicalis and C. parapsilosis respectively, Table 4, Additional file 4).

A high proportion (48\%) of the clusters identified contain only two genes and may not be biologically significant, as they appear at a high frequency in randomized data (see Methods). However, the metabolic clusters discussed here are highly significant, particularly for the three pathways discussed below.

\section{(i) The biotin biosynthesis pathway}

Biotin or vitamin $\mathrm{H}$ acts as a cofactor for a set of enzymes that catalyze carboxylation, decarboxylation, and transcarboxylation reactions in a number of crucial metabolic processes [48]. Most multicellular eukaryotes (except for plants) are biotin auxotrophs, whereas many bacterial species and some fungi (including Aspergillus and Saccharomyces species) are biotin prototrophs [49,50].

In $S$. cerevisiae 6 genes are involved in the production of biotin (BIO1-6). These are located in 2 clusters (BIO1/ $B I O 6$ and $B I O 3 / B I O 4 / B I O 5$, with $B I O 2$ at a different location) [49]. Hall and Dietrich [49] showed that the original eukaryotic biotin pathway was lost in the last common ancestor of Candida and Saccharomyces species, but it has been rebuilt through horizontal gene transfer from bacterial species via transfers of $B I O 3$ from $\delta$-proteobac- teria and $\mathrm{BIO} 4$ from $\alpha$-proteobacteria, followed by gene duplication and neofunctionalization.

We identified a biotin cluster of four genes (orthologs of S. cerevisiae BIO2, BIO3, $\mathrm{BIO} 4$ and $\mathrm{BIO5}$ ) in both $\mathrm{C}$. albicans strains (Figure 4). There is however an inversion of the surrounding region between SC5314 and WO-1 (Figure 4); this appears to result from a rearrangement between two members of the oligopeptide transporter gene family, OPT9 (a pseudogene) and OPT1.

The cluster in $C$. albicans is larger than the equivalent region in S. cerevisiae as it includes BIO2. BIO2 orthologs are in the same chromosomal region in $C$. albicans, $D$. hansenii, C. lusitaniae and C. guilliermondii (Figure 4). $B I O 3, B I O 4$ and $B I O 5$ are also adjacent to each other in $C$. tropicalis, and they appear to have been recruited to the $\mathrm{BIO} 2$ region in $\mathrm{C}$. albicans. Almost the entire cluster, together with an adjacent $O P T$ gene, is missing from $C$. dubliniensis. Only BIO2 remains, and this is located elsewhere in the genome. The absence of the biotin cluster in C. dubliniensis has previously been reported, and it was suggested that its presence in C. albicans may contribute to increased prevalence and virulence [51]. The entire set of $B I O$ genes is also absent from C. parapsilosis and $L$. elongisporus, and was probably lost in their last common ancestor (not shown). There is some conservation of synteny of the surrounding genes (not shown), suggesting the genes were lost together, as a cluster. Unlike S. cerevisiae, the biotin clusters in C. albicans and C. tropicalis are not sub-telomeric.

The remaining Candida species contain some genes involved in biotin synthesis. BIO2 is present in almost all species, suggesting it may play a role independent of biotin synthesis. $\mathrm{BIO} 4$ and $\mathrm{BIO} 5$ are clustered in $D$. hansenii with $\mathrm{BIO} 2$ elsewhere in the genome, whereas $\mathrm{BIO} 2, \mathrm{BIO} 3$ and $\mathrm{BIO} 4$ are present in P. stipitis, but are not clustered (not shown). It is not clear why some components of the

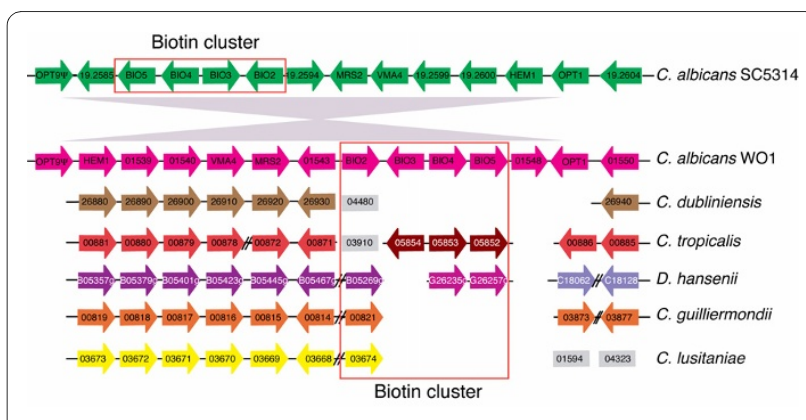

Figure $4 \mathrm{Gene}$ order around the biotin cluster. The diagram is redrawn from CGOB with some genes and species omitted for clarity. Blocks of color represent chromosomes. Homologs are organized in pillars. Changes in color indicate breaks in synteny. The grey triangles indicate an inversion between the C. albicans SC5314 and WO- 1 isolates. Diagonal lines indicate local inversions. Genes shown in grey boxes are not adjacent to any other gene shown. 
pathway are retained in some species. However, it may enable them to make biotin from some intermediates, as was described for $S$. cerevisiae [52]. It is generally assumed however that clustering of genes in biosynthetic pathways is the result of selection against toxic intermediates produced by incomplete pathways [49]. It is likely that the ancestral Candida species was able to synthesize biotin, but there has been substantial gene loss in many species.

In $S$. cerevisiae BIO6 is believed to have arisen through gene duplication of $\mathrm{BIO} 3$ followed by subfunctionalization [49]. We cannot locate an ortholog of BIO6 in any Candida species. Similarly we cannot locate any Candida ortholog of BIO1 (pimeloyl-CoA synthetase), the first enzyme involved in synthesizing biotin from pimelic acid. In $S$. cerevisiae $\mathrm{S} 288 \mathrm{C}, \mathrm{BIO} 1$ and $\mathrm{BIO6}$ are pseudogenes [49], but there is no evidence of corresponding pseudogenes in any Candida species. It is therefore unlikely that the genes are present in other unsequenced isolates of the same species.

The CGD biotin pathway data suggests that orf19.3567 (BIO32) is involved in biotin synthesis. BIO32 has a top BLASTP hit to $\mathrm{BIO} 3$ in S. cerevisiae. However, $\mathrm{BIO}$ belongs to a multigene family that also contains $A R G 8$, $C A R 2$ and UGA1. To determine the origin of BIO32 we reconstructed a phylogenetic tree using the same sequences used by Hall and Dietrich [49], and included ARG8, CAR2 and UGA1 from C. albicans and S. cerevisiae. Our phylogeny places the $S$. cerevisiae and C. albicans $\mathrm{BIO} 3$ orthologs together with bacterial sequences, indicating that they originated from horizontal gene transfer as suggested by Hall and Dietrich [49]. S. cerevisiae BIO6 is also grouped in this clade, supporting the hypothesis that it is a duplicate of $\mathrm{BIO}$. However, $\mathrm{BIO} 32$ from C. albicans is grouped with $S$. cerevisiae and C. albicans orthologs of $A R G 8, C A R 2$ and UGA1 in a separate clade (not shown). BIO32 is therefore most likely a duplicate of one of these genes, and is more likely to be involved in arginine or glutamate metabolism than in biotin synthesis.

\section{(ii) The $\mathrm{N}$-acetylglucosamine regulon}

It has been proposed that the ability of pathogenic strains of Candida to utilize sugars such as glucosamine and $N$ acetylglucosamine (Nag) as alternative carbon sources are important virulence factors [53]. C. albicans mutants incapable of utilizing $\mathrm{Nag}$ are less virulent in a murine model of systemic candidiasis compared to wild type isolates [54]. The three genes involved in the conversion of Nag to fructose-6-phosphate encode hexokinase kinase (HXK1/orf19.2154), Nag-6-phosphate deaminase (NAG1/orf19.2156) and Nag-6-phosphate deacetylase (DAC1/orf19.2157). These act sequentially on Nag and are present in C. albicans in a cluster termed the Nag regulon [53].
Our clustering analysis shows that the Nag regulon is conserved in all Candida species, with the exception of $C$. lusitaniae (Figure 5, Additional file 4). In the latter species, there has been an insertion of 5 species-specific genes in the region between $H X K 1$ and NAG1, resulting in a sequence of 19,931 basepairs (bp), whereas the intergenic region in the other species is less than $516 \mathrm{bp}$. Several of the inserted genes encode members of a family of cell wall genes, related to Flo1 from S. cerevisiae. The Nag cluster is sub-telomeric in many of the Candida species, and repeats of cell wall genes are commonly found near telomeres [55]. The conservation of the Nag regulon in pathogens like $C$. albicans and nonpathogens such as $P$. stipitis suggests that the ability to utilize Nag is not a virulence factor. NAG3, NAG4 and NAG6, which lie close to the NAG cluster in many Candida species (Figure 5), are not involved in the conversion of Nag, but are more likely to encode drug efflux pumps [56,57]. NAG3 is a tandem duplicate of NAG4 (Additional file 3, cluster 59), which occurred in the ancestor of C. albicans, C. dubliniensis, $C$. tropicalis, C. parapsilosis and L. elongisporus (Figure 1).

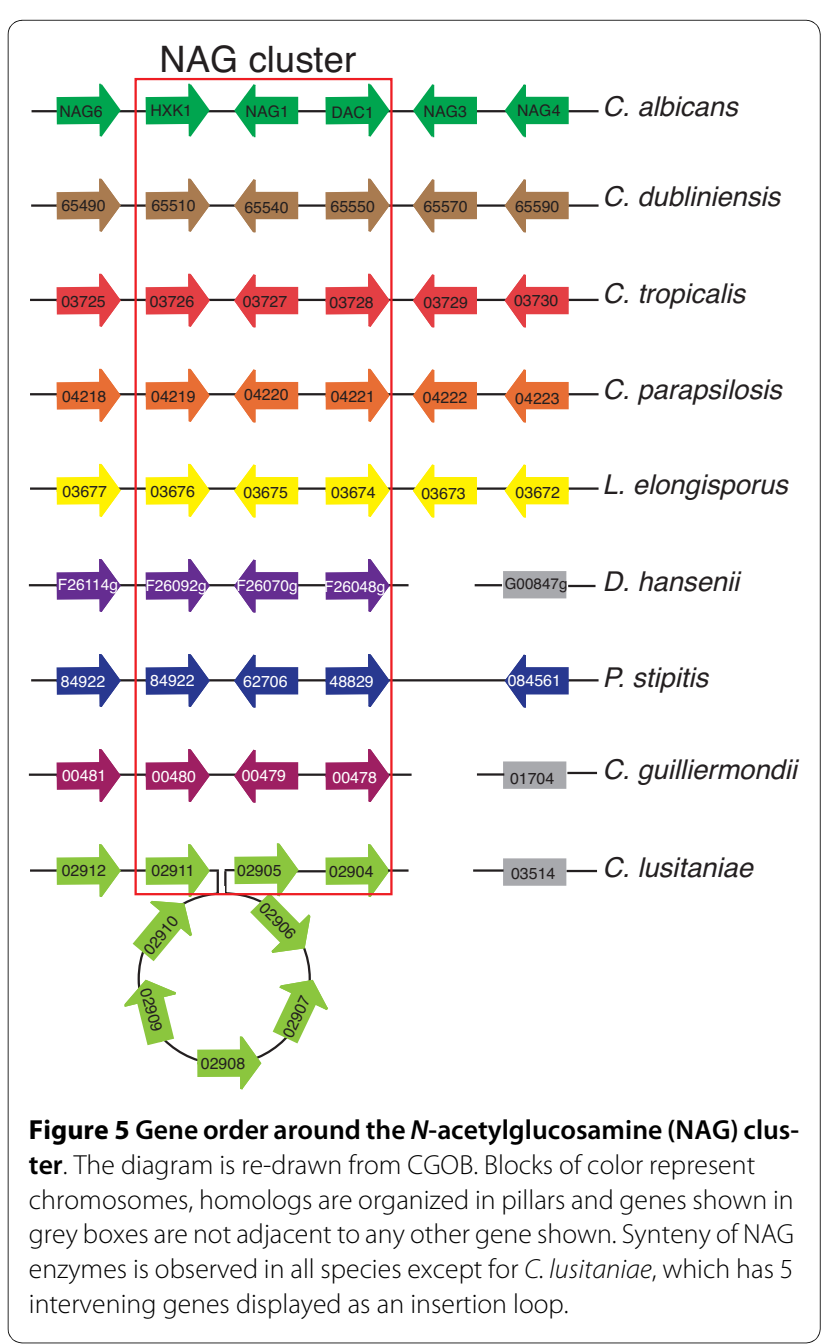


The phylogenomic distribution of the Nag regulon is intriguing. The cluster is found in the Candida species, but NAG1 and DAC1 are missing in the Saccharomyces lineage. Homologs are also absent from Ashbya gossypii and Kluyveromyces waltii, suggesting the cluster is missing from the entire Saccharomycetes lineage. However, the origin of the NAG cluster may be an ancient event. $D A C 1$ and NAG1 are in close proximity (within two genes) in the Aspergilli and in Neurospora crassa, which belong to the Pezizomycotina, a sister clade to the Saccharomycotina. DAC1 and NAG1 also lie within 2 genes in the Basidiomycete, Ustilago maydis. If the cluster arose in an ancestor of the Ascomycota and the Basidiomycota, it is very ancient, and the genes have been subsequently lost from many species (including Schizosaccharomyces).

\section{(iii) The Leloir galactose utilization pathway}

Galactose is utilized by most organisms through its conversion to glucose-6-phosphate, which then enters glycolysis [58]. The GAL pathway is composed of both structural and regulator elements [59]. The galactose metabolism structural genes of S. cerevisiae and C. albicans are well conserved, whereas their regulatory components are distinct [59]. In C. albicans the structural genes (GAL1, GAL10 and GAL7) are arranged in a cluster close to a hexose transporter HGT2 [59]. This cluster, together with two additional uncharacterized genes which lie between GAL10 and GAL7, is conserved in C. albicans, C. dubliniensis, C. parapsilosis and D. hansenii [59]. We show that the GAL pathway cluster is conserved in all Candida species present in CGOB (Figure 6).

Both $C$. albicans strains and C. lusitaniae have a gene insertion between GAL1 and GAL10 (not shown). The $C$. albicans gene (orf19.3671) is designated "dubious " by CGD, and is a pseudogene in WO-1, with no significant similarity to any other gene known from any other organism. Similarly the C. lusitaniae gene (CLUG_02293) has no significant homologs in either GenBank or CGOB Blast databases. The intergenic regions between GAL1 and GAL10 are 490 and 1362 nucleotides in C. lusitaniae and C. albicans, similar to the intergenic regions in all the other Candida species. It is likely therefore that both orf19.3671 and CLUG_02293 are errors in annotation, rather than real genes and so are not shown in Figure 6.

Expression of the hexose transporter HGT2 is strongly induced by galactose in C. albicans [59]. An ortholog of HGT2 is also very close to GAL1 in C. dubliniensis, C. tropicalis, C. guilliermondii and C. lusitaniae (Figure 6). HGT2 belongs to a large gene family, and while multiple homologs were located in many species, there is no family member adjacent to the GAL cluster in C. parapsilosis, L. elongisporus and P. stipitis. A putative ortholog in $D$. hansenii was identified, although it resides on a different chromosome to the GAL genes (Figure 6). It is possible

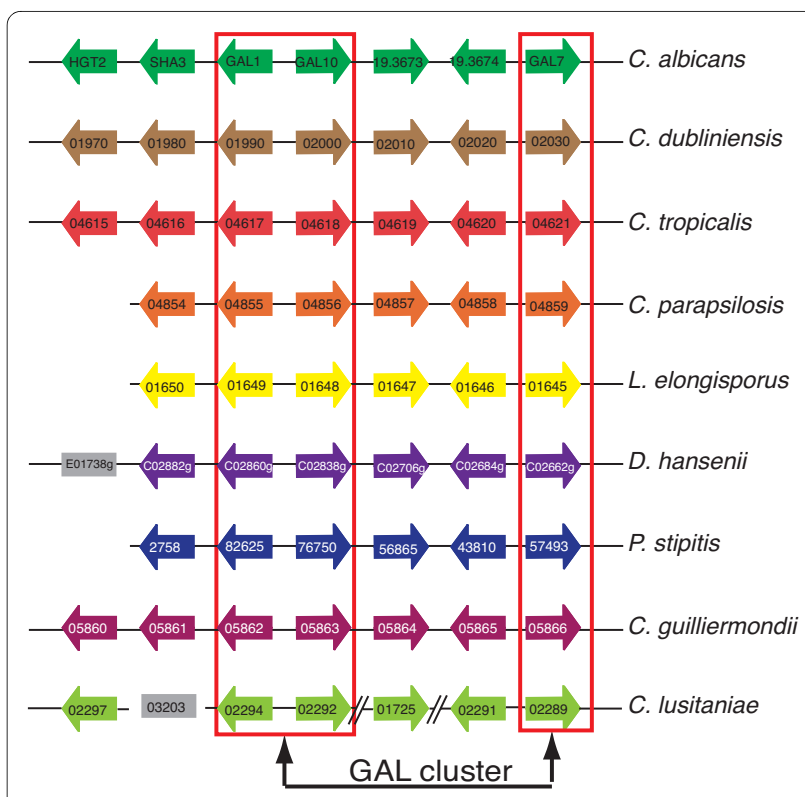

Figure 6 Gene order around the Galactose (GAL) cluster. The diagram is re-drawn from CGOB. Blocks of color represent chromosomes and homologs are organized in pillars. Diagonal lines indicate local inversions. Genes shown in grey boxes are not adjacent to any other gene shown. Dubious genes (orf19.3671 and CLUG_02293) from C. albicans and C. lusitaniae are not displayed.

that even though the relative position of the hexose transporter is not conserved, co-expression with the GAL genes may be.

Interestingly, orf19.3674, which lies between GAL10 and GAL7, appears to be a paralog of GAL10, and is conserved in all the Candida species. This was also noted in the P. stipitis genome [3]. Gal10 is more than twice the size of orf19.3674 (675 vs. 320 amino acids), and contains two recognized protein domains, an NAD dependent epimerase/dehydratase domain and an aldose 1-epimerase domain. Only the first domain is present in orf19.3674 and its orthologs. Expression of this gene is not influenced by galactose in C. albicans [59]. orf19.3674 may therefore have undergone subfunctionalization after duplication. Alternatively, recombination between a GAL10 precursor and another gene may have led to gene with a novel function. An ortholog of the adjacent conserved gene (orf19.3673) encodes a subunit of the transport protein particle (TRAPP) of the cis-Golgi in $S$. cerevisiae [60] and is unlikely to be involved in galactose metabolism.

\section{KEGG Analysis}

We also assigned genes in each Candida species to individual metabolic pathways using the Kyoto Encyclopedia of Genes and Genomes (KEGG) [61]. This approach permitted us to investigate species-specific metabolic pathways, as well as pathways that have been described only 
in C. albicans SC5314. Approximately 200 metabolic pathways were reconstructed for each Candida species (Table 5). However, $\sim 30 \%$ of these were redundant (Table 5). For example, in C. dubliniensis, the inferred components of the pathways for peptidoglycan and alkaloid biosynthesis are completely contained in the alanine, aspartate and glutamate metabolism pathway (not shown). The number of gene assignments to the nonredundant pathways is approximately 2000 for each Candida species. On average close to $50 \%$ of these are represented in multiple pathways (Table 5). Therefore between 16-19\% of genes from each Candida species have been successfully assigned to a unique KEGG metabolic pathway (Table 5), equating to $17.8 \%$ of all Candida genes represented in CGOB.

We interrogated each Candida species in CGOB for evidence of clustering in the non-redundant KEGG pathways (Table 5). In total we identified 62 pathways; (33-44 per species) that display some evidence of gene clustering (Table 5). There are 767 KEGG clusters (KCs) shared amongst all species, and of these 32 have arisen through tandem duplication (Additional file 5). Most of the identified KCs are small, containing two or three genes. A high proportion (75\%) of these may not be biologically significant (Additional file 4), as they appear at a high frequency in randomized data (see Methods).

Overall the observed KEGG metabolic pathway clusters are generally distinct from those located using the CGD pathways (Additional file 4 and Additional file 5). There is a small degree of crossover, including the CGD tRNA charging pathway, which is analogous to KEGG's aminoacyl-tRNA biosynthesis pathway, the CGD aerobic respiration pathway which is equivalent to oxidative phosphorylation in KEGG, and the galactose metabolism and histidine metabolism pathways in both. Several pathways (such as clustering of histone protein genes (ko05322) and ribosomal protein genes (ko03010) have been described previously. It is likely that other clusters will be identified when the assignments to pathways improve. For example, Jeffries and Van Vleet [26] identified some small clusters of functionally-related genes in $P$. stipitis by visual inspection. Our approach found some of these, but not all.

\section{Conclusions}

We describe here a unique tool for studying evolution and gene function in Candida species. During the development of CGOB we improved the existing annotations for several species, by identifying and removing partial open reading frames, and by manually assigning homology, based on sequence similarity and synteny. We also provide a detailed analysis of gene clusters in Candida, which will provide a basis for future investigation. We identified many of the clusters described in only one species $[3,4,26,53,59]$. However, we have also shown the benefits of a comparative approach; some clusters (such as NAG) although originally described in C. albicans only are present in all Candida species, whereas others (such as CIP) are unique to one (P. stiptis). Our analysis provides an important resource that is now available for the Candida community.

\section{Methods}

\section{Genome Data}

The complete C. albicans (SC5314) genome (Assembly 21 [31]) was obtained from the Candida genome database (CGD) [62]. Gene sets for C. albicans WO-1, C. tropicalis, L. elongisporus, C. guilliermondii, and C. lusitaniae [5] were obtained directly from the Broad Institute [63] and for C. dubliniensis [7] from GeneDB at the Wellcome Trust Sanger Institute [64]. The first assembly of the C. parapsilosis genome was downloaded from the Sanger Institute [65] and in-house gene annotations were called (as described in Fitzpatrick et al [8]). The resultant gene set contains 5,809 protein-coding genes. The $C$. parapsilosis genome was also automatically annotated by

Table 5: KEGG metabolic pathways that show evidence of gene clustering in Candida species.

\begin{tabular}{|c|c|c|c|c|c|}
\hline Species & Pathways & $\begin{array}{c}\text { Non-Redundant } \\
\text { pathways }\end{array}$ & $\begin{array}{l}\text { Genes in } \\
\text { Pathways }\end{array}$ & $\begin{array}{l}\text { Unique Genes } \\
\text { in Pathways }\end{array}$ & $\begin{array}{c}\text { Metabolic } \\
\text { clusters }\end{array}$ \\
\hline C. albicans & 190 & 136 & 1870 & 991 (16.0\%) & 38 \\
\hline C. dubliniensis & 196 & 139 & 1857 & $968(16.3 \%)$ & 39 \\
\hline C. tropicalis & 201 & 139 & 1864 & $988(15.9 \%)$ & 35 \\
\hline C. parapsilosis & 204 & 149 & 2026 & 1062 (18.3\%) & 34 \\
\hline L. elongisporus & 202 & 142 & 1991 & $1048(18.4 \%)$ & 33 \\
\hline D. hansenii & 209 & 152 & 2165 & $1148(18.2 \%)$ & 39 \\
\hline P. stipitis & 205 & 151 & 2134 & $1114(19.1 \%)$ & 44 \\
\hline C. guilliermondii & 210 & 158 & 2162 & $1126(19.3 \%)$ & 36 \\
\hline C. Iusitaniae & 207 & 146 & 2069 & 1091 (18.6\%) & 34 \\
\hline
\end{tabular}


the Broad Institute [5], and we use these gene names where possible.

\section{Phylogenetic relationships}

Phylogenetic relationships were determined using a supertree approach. All ten Candida genomes as well as two outgroups (Saccharomyces cerevisiae and Candida glabrata) were merged into a local Blast database. For a full descriptions of the methodology used please refer to Fitzpatrick et al [8].

\section{Homology pillars and genome editing}

Sets of homologous genes are stored in CGOB's pillars (Figure 2). Pillars are the core data structures used to store homology assignments across all species [20]. All genes were integrated into homology pillars by performing an automated bi-directional best BLASTP hit (Evalue cut-off of $10^{-5}$ ) strategy against $C$. albicans SC5314. A second round of automated searching merged singleton pillars using a BLASTP hit (E- value cut-off of $10^{-5}$ ) and synteny with at least one gene in an adjacent pillar. We then systematically manually edited CGOB by browsing along each Candida chromosome validating and refining homology pillars.

Several potential genes in the automatically called open reading frames sets are incomplete or "partial". We merged partial ORFs where possible, by aligning them against their complete orthologs from the other Candida genomes using Muscle [66]. The resultant alignments were manually checked and where appropriate, partial ORFs were merged and the resulting gene models were renamed, and added to CGOB's pillars. For completeness both the merged genes and the original partial ORFs have been retained in the CGOB Blast sequence database.

\section{Duplications}

Genes that have arisen through tandem duplication were located using bl2seq from the NCBI suite of Blast executables. A tandem repeat was defined as adjacent genes with an E- value cut-off of $10^{-10}$ with a highest scoring sequence pair (HSP) more than half the length of the shortest sequence. This approach filters out genes with similarity over short regions. Tandem genes that are evolving rapidly or have low sequence complexity may not be located using sequence similarity. We therefore programmed CGOB to compare tandem duplicates in all genomes, and used synteny to locate fast evolving tandems (or tandems with low complexity) in another genome.

Synonymous $\left(\mathrm{d}_{\mathrm{S}}\right)$ and nonsynonymous $\left(\mathrm{d}_{\mathrm{N}}\right)$ substitution rates for genes located in tandem clusters were estimated using the methods of Yang and Nielsen [67] as implemented in yn00 in the PAML suite [68].
To identify multigene families, every gene in a particular Candida proteome was searched against every other gene in its cognate genome. Genes with a BLASTP Evalue less than $10^{-30}$ and a HSP more than $60 \%$ the length of the shortest sequence were considered to be members of the same family, this is the same strategy used by Braun et al [31].

\section{Locating clusters of adjacent genes in metabolic pathways} Metabolic pathways for C. albicans SC5314 were downloaded from the Candida Genome Database [62]. The gene identifiers for each enzymatic step were mapped on CGOB. Clusters were defined as identifiers belonging to a particular metabolic pathway that lie within a contiguous window of 10 genes. The presence or absences of C. albicans SC5314 pathway homologs were then scored in the remaining nine Candida genomes.

For completeness we automatically inferred individual metabolic pathways for all Candida species using the KEGG automatic annotation server (KAAS) [69]. KAAS is based on reciprocally best BLAST similarity hits against all KEGG orthology (KO) groups of functionally related genes assigned in the KEGG GENES database. KAAS assigned each Candida gene a KO number and these were subsequently mapped to one of KEGG's reference metabolic pathways. All Candida KO identifiers were mapped onto $\mathrm{CGOB}$ and we searched for metabolic clusters as described above.

The significance of metabolic clusters was tested using simulations where gene order was randomized to give pseudogenomes. Both CGD and KEGG pathway components were mapped onto randomized genome data and scored as described above. This process was repeated 10000 times for each pathway in each Candida genome. Clusters are considered significant if the number of linked genes in the pseudogenome is less than that observed in the real genome $95 \%$ of the time.

\section{Additional material}

Additional file 1 Merging partial open reading frames. Section of alignment illustrating that the original automatically called gene sets contained partial open reading frames. In this example LELG_01496 and LELG_01495 from L. elongisporus are merged to give a new single gene (LELG_01496*)

Additional file $\mathbf{2}$ List of partial ORFs in datasets obtained from sequencing centers. Merged genes all have $\mathrm{a}^{*}$ suffix and are present in CGOB. Partial ORFs have been removed from CGOB pillars but are present in the CGOB Blast database.

Additional file 3 List of all tandem duplicates located by CGOB. a) Clusters are labeled 1-502. Those with $a d_{N} / d_{S}$ value $>1$ are highlighted in red. Clusters displaying a $\wedge$ indicate that the initial BLAST search strategy failed to infer homology. Clusters displaying * indicate that there are inter vening genes but they may be spurious gene models. Clusters displaying (INS) indicate that there is one intervening gene. b) List of tandem clusters with $a d_{N} / d_{S}>1$. 
Additional file 4 List of CGD pathways and the corresponding genes that display evidence of clustering in each Candida species. Numbers in parenthesis refer to cluster numbers and are retained across species. Clusters with "sig" in parenthesis infer that the cluster is significantly better than randomized data.

Additional file $\mathbf{5}$ List of KEGG pathways and the corresponding genes that display evidence of clustering in each Candida species. Clusters have been assigned numbers (KC) so it is possible to locate a cluster present in one species that is absent in another. Clusters denoted with a TD infer that the cluster has arisen through tandem duplication. Clusters that are significantly better than randomized data are highlighted with purple shading.

\section{Abbreviations}

CGD: Candida genome database; CGOB: Candida Gene Order Browser; YGOB Yeast Gene Order Browser; HSP: highest scoring sequence pair; KEGG: Kyoto Encyclopedia of Genes and Genomes; KCs: KEGG clusters; KAAS: KEGG automatic annotation server; KO: KEGG orthology; ORF: open reading frame; PIPKc: Phosphatidylinositol Phosphate Kinase; Nag: N-acetylglucosamine; HXK1: hexokinase kinase; DAC1: Nag-6-phosphate deacetylase; bp: basepairs; $\mathbf{d}_{\mathbf{s}}$ : synonymous substitution; $\mathbf{d}_{\mathbf{N}}$ : nonsynonymous substitution.

\section{Authors' contributions}

DAF and GB were involved in the design phase. KPB developed and installed software. POG installed software. POG and DAF sourced homologs. DAF merged partial genes and manually curated homology columns. DAF and GB examined synteny, duplication and cluster data. DAF and GB drafted the manuscript. All authors read and approved the final manuscript.

\section{Acknowledgements}

We would like to acknowledge the financial support of the Health Research Board of Ireland and Science Foundation Ireland (SFI 08/I.1/B1865), and the assistance of Ken Wolfe in adapting YGOB and for reading the manuscript.

\section{Author Details}

1 UCD School of Biomolecular and Biomedical Science, Conway Institute, University College Dublin, Belfield, Dublin 4, Ireland, 2Department of Biology, The National University of Ireland, Maynooth, County Kildare, Ireland, ${ }^{3}$ UCD School of Medicine and Medical Science, Conway Institute, University College Dublin, Belfield, Dublin 4, Ireland and 4 Smurfit Institute of Genetics, University of Dublin, Trinity College Dublin, Dublin 2, Ireland

Received: 21 December 2009 Accepted: 10 May 2010 Published: 10 May 2010

\section{References}

1. Wisplinghoff $H$, Bischoff T, Tallent SM, Seifert H, Wenzel RP, Edmond MB: Nosocomial bloodstream infections in US hospitals: analysis of 24,179 cases from a prospective nationwide surveillance study. Clin Infect Dis 2004, 39:309-317.

2. Pfaller MA, Diekema DJ: Epidemiology of invasive candidiasis: a persistent public health problem. Clin Microbiol Rev 2007, 20:133-163.

3. Jeffries TW, Grigoriev IV, Grimwood J, Laplaza JM, Aerts A, Salamov A, Schmutz J, Lindquist E, Dehal P, Shapiro H, Jin YS, Passoth V, Richardson PM: Genome sequence of the lignocellulose-bioconverting and xylosefermenting yeast Pichia stipitis. Nat Biotechno/ 2007, 25:319-326.

4. Dujon B, Sherman D, Fischer G, Durrens P, Casaregola S, Lafontaine I, De Montigny J, Marck C, Neuveglise C, Talla E, Goffard N, Frangeul L, Aigle M, Anthouard V, Babour A, Barbe V, Barnay S, Blanchin S, Beckerich JM, Beyne E, Bleykasten C, Boisrame A, Boyer J, Cattolico L, Confanioleri F, De Daruvar A, Despons L, Fabre E, Fairhead C, Ferry-Dumazet H, Groppi A, Hantraye F, Hennequin $C$, Jauniaux $N$, Joyet $P$, Kachouri R, Kerrest A, Koszul R, Lemaire M, Lesur I, Ma L, Muller H, Nicaud JM, Nikolski M, Oztas S, OzierKalogeropoulos O, Pellenz S, Potier S, Richard GF, Straub ML, Suleau A, Swennen D, Tekaia F, Wesolowski-Louvel M, Westhof E, Wirth B, ZeniouMeyer M, Zivanovic I, Bolotin-Fukuhara M, Thierry A, Bouchier C, Caudron B, Scarpelli C, Gaillardin C, Weissenbach J, Wincker P, Souciet JL: Genome evolution in yeasts. Nature 2004, 430:35-44

5. Butler G, Rasmussen MD, Lin MF, Santos MA, Sakthikumar S, Munro CA Rheinbay E, Grabherr M, Forche A, Reedy JL, Agrafioti I, Arnaud MB, Bates
S, Brown AJ, Brunke S, Costanzo MC, Fitzpatrick DA, de Groot PW, Harris D, Hoyer LL, Hube B, Klis FM, Kodira C, Lennard N, Logue ME, Martin R, Neiman AM, Nikolaou E, Quail MA, Quinn J, Santos MC, Schmitzberger FF, Sherlock G, Shah P, Silverstein KA, Skrzypek MS, Soll D, Staggs R, Stansfield I, Stumpf MP, Sudbery PE, Srikantha T, Zeng Q, Berman J, Berriman M, Heitman J, Gow NA, Lorenz MC, Birren BW, Kellis M, Cuomo CA: Evolution of pathogenicity and sexual reproduction in eight Candida genomes. Nature 2009, 4;459(7247):657-62.

6. Jones T, Federspiel NA, Chibana H, Dungan J, Kalman S, Magee BB, Newport G, Thorstenson YR, Agabian N, Magee PT, Davis RW, Scherer S: The diploid genome sequence of Candida albicans. Proc Natl Acad Sci USA 2004, 101:7329-7334

7. Jackson AP, Gamble JA, Yeomans T, Moran GP, Saunders D, Harris D, Aslett M, Barrell JF, Butler G, Citiulo F, Coleman DC, de Groot PW, Goodwin TJ Quail MA, McQuillan J, Munro CA, Pain A, Poulter RT, Rajandream MA, Renauld H, Spiering MJ, Tivey A, Gow NA, Barrell B, Sullivan DJ, Berriman $\mathrm{M}$ : Comparative genomics of the fungal pathogens Candida dubliniensis and C. albicans. Genome Res 2009 in press.

8. Fitzpatrick DA, Logue ME, Stajich JE, Butler G: A Fungal phylogeny based on 42 complete genomes derived from supertree and combined gene analysis. BMC Evol Biol 2006, 6:99.

9. Sugita T, Nakase T: Non-universal usage of the leucine CUG codon and the molecular phylogeny of the genus Candida. Syst App/Microbiol 1999, 22:79-86.

10. Fitzpatrick DA, Logue ME, Butler G: Evidence of recent interkingdom horizontal gene transfer between bacteria and Candida parapsilosis. BMC Evol Biol 2008, 8:181.

11. Almeida RS, Brunke S, Albrecht A, Thewes S, Laue M, Edwards JE, Filler SG, Hube B: The hyphal-associated adhesin and invasin Als3 of Candida albicans mediates iron acquisition from host ferritin. PLOS Pathog 2008, 4:e1000217.

12. Hoyer LL, Green CB, Oh SH, Zhao X: Discovering the secrets of the Candida albicans agglutinin-like sequence (ALS) gene family--a sticky pursuit. Med Mycol 2008, 46:1-15.

13. Yeater KM, Chandra J, Cheng G, Mukherjee PK, Zhao X, Rodriguez-Zas SL, Kwast KE, Ghannoum MA, Hoyer LL: Temporal analysis of Candida albicans gene expression during biofilm development. Microbiology 2007, 153:2373-2385.

14. De Groot PW, Hellingwerf KJ, Klis FM: Genome-wide identification of fungal GPI proteins. Yeast 2003, 20:781-796.

15. Bailey DA, Feldmann PJ, Bovey M, Gow NA, Brown AJ: The Candida albicans HYR1 gene, which is activated in response to hyphal development, belongs to a gene family encoding yeast cell wall proteins. J Bacteriol 1996, 178:5353-5360.

16. Rossignol T, Lechat $P$, Cuomo C, Zeng Q, Moszer I, d'Enfert C: CandidaDB: a multi-genome database for Candida species and related Saccharomycotina. Nucleic Acids Res 2008, 36:D557-561.

17. Arnaud MB, Costanzo MC, Skrzypek MS, Shah P, Binkley G, Lane C, Miyasato SR, Sherlock G: Sequence resources at the Candida Genome Database. Nucleic Acids Res 2007, 35:D452-456

18. CGOB [http://cgob.ucd.ie/]

19. Byrne KP, Wolfe KH: Visualizing syntenic relationships among the hemiascomycetes with the Yeast Gene Order Browser. Nucleic Acids Res 2006, 34:D452-455.

20. Byrne KP, Wolfe KH: The Yeast Gene Order Browser: combining curated homology and syntenic context reveals gene fate in polyploid species. Genome Res 2005, 15:1456-1461.

21. Hong EL, Balakrishnan R, Dong Q, Christie KR, Park J, Binkley G, Costanzo MC, Dwight SS, Engel SR, Fisk DG, Hirschman JE, Hitz BC, Krieger CJ, Livstone MS, Miyasato SR, Nash RS, Oughtred R, Skrzypek MS, Weng S, Wong ED, Zhu KK, Dolinski K, Botstein D, Cherry JM: Gene Ontology annotations at SGD: new data sources and annotation methods. Nucleic Acids Res 2008, 36:D577-581.

22. Jones JM, Nau K, Geraghty MT, Erdmann R, Gould SJ: Identification of peroxisomal acyl-CoA thioesterases in yeast and humans. J Biol Chem 1999, 274:9216-9223.

23. Lorenz MC, Bender JA, Fink GR: Transcriptional response of Candida albicans upon internalization by macrophages. Eukaryot Cell 2004, 3:1076-1087.

24. Piekarska K, Mol E, Berg M van den, Hardy G, Burg J van den, van Roermund C, MacCallum D, Odds F, Distel B: Peroxisomal fatty acid beta- 
oxidation is not essential for virulence of Candida albicans. Eukaryot Cell 2006, 5:1847-1856.

25. Palkova Z, Devaux F, Icicova M, Minarikova L, Le Crom S, Jacq C: Ammonia pulses and metabolic oscillations guide yeast colony development. Mol Bio/ Cell 2002, 13:3901-3914.

26. Jeffries TW, Van Vleet JR: Pichia stipitis genomics, transcriptomics, and gene clusters. FEMS Yeast Res 2009, 9:793-807.

27. Fan C, Chen Y, Long M: Recurrent tandem gene duplication gave rise to functionally divergent genes in Drosophila. Mol Biol Evol 2008, 25:1451-1458.

28. Emes RD, Yang Z: Duplicated paralogous genes subject to positive selection in the genome of Trypanosoma brucei. PLOS ONE 2008, 3:e2295.

29. Nantel A, Dignard D, Bachewich C, Harcus D, Marcil A, Bouin AP, Sensen $\mathrm{CW}$, Hogues $\mathrm{H}$, van het Hoog M, Gordon P, Rigby T, Benoit F, Tessier DC, Thomas DY, Whiteway M: Transcription profiling of Candida albicans cells undergoing the yeast-to-hyphal transition. Mol Biol Cell 2002, 13:3452-3465.

30. Friedman R, Hughes AL: Gene duplication and the structure of eukaryotic genomes. Genome Res 2001, 11:373-381.

31. Braun BR, van Het Hoog M, d'Enfert C, Martchenko M, Dungan J, Kuo A, Inglis DO, Uhl MA, Hogues H, Berriman M, Lorenz M, Levitin A, Oberholzer U, Bachewich C, Harcus D, Marcil A, Dignard D, louk T, Zito R, Frangeul L, Tekaia F, Rutherford K, Wang E, Munro CA, Bates S, Gow NA, Hoyer LL, Kohler G, Morschhauser J, Newport G, Znaidi S, Raymond M, Turcotte B, Sherlock G, Costanzo M, Ihmels J, Berman J, Sanglard D, Agabian N, Mitchell AP, Johnson AD, Whiteway M, Nantel A: A human-curated annotation of the Candida albicans genome. PLoS Genet 2005, 1:36-57.

32. Wolfe KH, Shields DC: Molecular evidence for an ancient duplication of the entire yeast genome. Nature 1997, 387:708-713.

33. Tirosh I, Barkai N: Comparative analysis indicates regulatory neofunctionalization of yeast duplicates. Genome Bio/ 2007, 8:R50

34. Bon E, Casaregola S, Blandin G, Llorente B, Neuveglise C, Munsterkotter M, Guldener U, Mewes HW, Van Helden J, Dujon B, Gaillardin C: Molecular evolution of eukaryotic genomes: hemiascomycetous yeast spliceosomal introns. Nucleic Acids Res 2003, 31:1121-1135.

35. Derr LK, Strathern JN: A role for reverse transcripts in gene conversion. Nature 1993, 361:170-173

36. Stajich JE, Dietrich FS: Evidence of mRNA-mediated intron loss in the human-pathogenic fungus Cryptococcus neoformans. Eukaryot Cell 2006, 5:789-793.

37. Mitrovich QM, Tuch BB, Guthrie C, Johnson AD: Computational and experimental approaches double the number of known introns in the pathogenic yeast Candida albicans. Genome Res 2007, 17:492-502.

38. Fan J, Chaturvedi V, Shen SH: Identification and phylogenetic analysis of a glucose transporter gene family from the human pathogenic yeast Candida albicans. J Mol Evol 2002, 55:336-346.

39. Roy SW, Penny D: On the incidence of intron loss and gain in paralogous gene families. Mol Biol Evol 2007, 24:1579-1581.

40. Martinez-Lopez R, Park H, Myers CL, Gil C, Filler SG: Candida albicans Ecm33p is important for normal cell wall architecture and interactions with host cells. Eukaryot Cell 2006, 5:140-147.

41. Zorio DA, Cheng NN, Blumenthal T, Spieth J: Operons as a common form of chromosomal organization in C. elegans. Nature 1994, 372:270-272.

42. Blumenthal T: Gene clusters and polycistronic transcription in eukaryotes. Bioessays 1998, 20:480-487.

43. Blumenthal T, Evans D, Link CD, Guffanti A, Lawson D, Thierry-Mieg J, Thierry-Mieg D, Chiu WL, Duke K, Kiraly M, Kim SK: A global analysis of Caenorhabditis elegans operons. Nature 2002, 417:851-854.

44. Keller NP, Hohn TM: Metabolic Pathway Gene Clusters in Filamentous Fungi. Fungal Genet Biol 1997, 21:17-29.

45. Hittinger CT, Rokas A, Carroll SB: Parallel inactivation of multiple GAL pathway genes and ecological diversification in yeasts. Proc Natl Acad Sci USA 2004, 101:14144-14149.

46. Wong $\mathrm{S}$, Wolfe $\mathrm{KH}$ : Birth of a metabolic gene cluster in yeast by adaptive gene relocation. Nat Genet 2005, 37:777-782.

47. Lee JM, Sonnhammer EL: Genomic gene clustering analysis of pathways in eukaryotes. Genome Res 2003, 13:875-882.

48. Knowles JR: The mechanism of biotin-dependent enzymes. Annu Rev Biochem 1989, 58:195-221.
49. Hall C, Dietrich FS: The Reacquisition of Biotin Prototrophy in Saccharomyces cerevisiae Involved Horizontal Gene Transfer, Gene Duplication and Gene Clustering. Genetics 2007, 177:2293-2307.

50. Shchelokova EV, Vorob'eva LI: [Biotin formation by the fungus Rhizopus delemar]. Prikl Biokhim Mikrobiol 1982, 18:630-635.

51. Moran G, Stokes C, Thewes S, Hube B, Coleman DC, Sullivan D: Comparative genomics using Candida albicans DNA microarrays reveals absence and divergence of virulence-associated genes in Candida dubliniensis. Microbiology 2004, 150:3363-3382.

52. Phalip V, Kuhn I, Lemoine Y, Jeltsch JM: Characterization of the biotin biosynthesis pathway in Saccharomyces cerevisiae and evidence for a cluster containing $\mathrm{BIO} 5$, a novel gene involved in vitamer uptake. Gene 1999, 232:43-51.

53. Kumar MJ, Jamaluddin MS, Natarajan K, Kaur D, Datta A: The inducible Nacetylglucosamine catabolic pathway gene cluster in Candida albicans: discrete $\mathrm{N}$-acetylglucosamine-inducible factors interact at the promoter of NAG1. Proc Natl Acad Sci USA 2000, 97:14218-14223.

54. Singh P, Ghosh S, Datta A: Attenuation of virulence and changes in morphology in Candida albicans by disruption of the $\mathrm{N}$ acetylglucosamine catabolic pathway. Infect Immun 2001, 69:7898-7903.

55. Teunissen AW, Steensma HY: Review: the dominant flocculation genes of Saccharomyces cerevisiae constitute a new subtelomeric gene family. Yeast 1995, 11:1001-1013.

56. Sengupta M, Datta A: Two membrane proteins located in the Nag regulon of Candida albicans confer multidrug resistance. Biochem Biophys Res Commun 2003, 301:1099-1108.

57. Yamada-Okabe T, Yamada-Okabe H: Characterization of the CaNAG3, CaNAG4, and CaNAG6 genes of the pathogenic fungus Candida albicans: possible involvement of these genes in the susceptibilities of cytotoxic agents. FEMS Microbiol Lett 2002, 212:15-21.

58. Leloir LF: The enzymatic transformation of uridine diphosphate glucose into a galactose derivative. Arch Biochem 1951, 33:186-190.

59. Martchenko M, Levitin A, Hogues H, Nantel A, Whiteway M: Transcriptional rewiring of fungal galactose-metabolism circuitry. Curr Biol 2007, 17:1007-1013.

60. Sacher M, Barrowman J, Schieltz D, Yates JR, Ferro-Novick S: Identification and characterization of five new subunits of TRAPP. Eur J Cell Biol 2000, 79:71-80.

61. Ogata H, Goto S, Sato K, Fujibuchi W, Bono H, Kanehisa M: KEGG: Kyoto Encyclopedia of Genes and Genomes. Nucleic Acids Res 1999, 27:29-34.

62. The Candida Genome Database [http://www.candidagenome.org]

63. The Candida group at the Broad Institute [http://www.broad.mit.edu/ annotation/genome/candida group/MultiHome.html]

64. GeneDB [http://www.genedb.org]

65. The Wellcome Trust Sanger Institute [http://www.sanger.ac.uk/]

66. Edgar RC: MUSCLE: multiple sequence alignment with high accuracy and high throughput. Nucleic Acids Res 2004, 32:1792-1797.

67. Yang Z, Nielsen R: Estimating synonymous and nonsynonymous substitution rates under realistic evolutionary models. Molecular Biology and Evolution 2000, 17:32-43.

68. Yang Z: PAML: a program package for phylogenetic analysis by maximum likelihood. Computer Applications in the Biosciences: Cabios 1997, 13:555-556

69. Moriya Y, Itoh M, Okuda S, Yoshizawa AC, Kanehisa M: KAAS: an automatic genome annotation and pathway reconstruction server. Nucleic Acids Res 2007, 35:W182-185.

doi: 10.1186/1471-2164-11-290

Cite this article as: Fitzpatrick et al., Analysis of gene evolution and metabolic pathways using the Candida Gene Order Browser BMC Genomics 2010 11:290 